\title{
ZUR ZOOGEOGRAPHIE DER ARU- UND KEI-INSELN
}

VON

\author{
H. MERTON \\ (HEIDELBERG)
}

Die Bearbeitung der zoologischen Ausbeute meiner, zusammen mit Dr. J. Roux 1907/08 unternommenen Forschungsreise, nach den Aru- und Kei-Inseln ist jetzt soweit fortgeschritten, dass die Resultate aller zur Beurteilung der Zoogeographie dieser Inseln wichtigen Gruppen vorliegen. Es ist daher jetzt möglich, über die Zusammensetzung der Faunen dieser beiden östlichsten Inselgruppen des Indo-australischen Archipels einen Ueberblick zu gewinnen, und durch Vergleichung Einblick zu erhalten in die Beziehungen dieser Faunen zueinander, zu den benachbarten grossen Landmassen von Neu-Guinea und Australien und $\mathrm{zu}$ dem westwärts ausgebreiteten inselreichen Archipel. Von der Land- und Süsswasserfauna können diejenigen Abteilungen unberücksichtigt bleiben, die wegen ihres hohen geologischen Alters und der weiten Verbreitung ihrer Arten zur Beurteilung zoogeographischer Fragen von geringer Bedeutung sind. Es sollen hier also hauptsächlich die Klassen der Wirbeltiere und die Land- und Süsswasser-Mollusken besprochen werden. Eine Zusammenfassung aller Ergebnisse der Reise ist als Abschluss der "Wissenschaftlichen Ergebnisse” in Aussicht genommen '),

Die Säugetiere, DE BEAUFORT ${ }^{2}$ ) hat sie bearbeitet, sind wenig auffallend im Landschaftsbild der beiden Inselgruppen; es sind grösstenteils kleine Formen, von denen die meisten eine nächtliche Lebensweise führen oder im Verborgenen leben, wie viele Nagetiere. Andere Arten sind in verhältnismässig geringer Individuenzahl vorhanden. Am häufigsten sieht man die Phalanger-Arten, die wenn auch am Tage sehr träge, doch öfters im Geäst der Bäume zu entdecken sind.

Von den 9 Arten von Beuteltieren der Aru- und Kei-Inseln haben 3 eine weitere Verbreitung und leben auch auf Neu-Guinea und im östlichen Teil des Archipels. Es sind das zwei Arten der Gattung Phalanger, die von allen Beuteltieren am weitesten westwärts (Celebes) vorgedrungen ist, und ein Petaurus. Zwei Beuteltier-Arten finden sich auf Aru, Kei und Neu-Guinea (davon ist allerdings Thylogale in einer sehr nahstehenden Art von Neu-Guinea bekannt, die andere Art ist ein Perameles). Nur diese 5 Beuteltierarten, alle mit etwas weiterem Verbreitungsgebiet, kommen auf den Kei-Inseln vor, sie alle sind auch, wie bemerkt, von den Aru Inseln bekannt. Auf Aru kommen ausserdem noch 4 Arten vor, die auch in Neu-Guinea gefunden worden sind. Die Beuteltier-Fauna der Kei-Inseln ist also merklich ärmer, als die der Aru-Inseln. Dafür leben auf den Kei-Inseln eine Carnivoren- und 2 Insectivoren-Arten (Paradoxurus und Crocidura $2 \mathrm{Sp}$.), die als indische Elemente $\mathrm{zu}$ betrachten wären, die aber wie DE BEAUFORT annimmt, wohl durch den Menschen eingeschleppt worden sind. Von 7 auf den beiden Inselgruppen lebenden Nagetieren sind 2 Arten als verschleppt, für diese Betrachtungen auszuschalten, die übrigen 5 gehören der australischen Region an. Zusammenfassend ist von den Marsupialiern und Rodentiern zu sagen, dass alle Arten, die beiden Inseln gemein sind, auch auf Neu-Guinea vorkommen. Zwei Säugetiere der Aru-Inseln, eine Cervus-Art und Sus papuensis sind vom Menschen eingeführt worden. Unberücksichtigt können auch die Chiropteren

1) Erscheint in Abhandl. Senckenberg. Naturf. Gesellsch. XXXV.

2) L. F. DE BEAUFORT, Die Säugetiere der Aru- und Kei-Inseln. Abhandl. Senckenberg. Naturf. Ges. XXXIV, 1911. 
bleiben, auf die mehr als die Hälfte aller für die beiden Inseln bekannten Säugetierè entfällt und die Pinnipedier.

Ein viel auffallenderes Element der Tierwelt der Aru- und Kei-Inseln bilden die Vögel, und überraschend ist die grosse Verschiedenheit in der Zusammensetzung der Avifauna der beiden Inselgruppen, obwohl beide etwa gleichweit von Neu-Guinea entfernt sind. Von den Aru-Inseln sind nach der Zusammenstellung von v. BERLEPSCH ${ }^{1}$ ) 191 Arten bekannt, von denen 4 Arten und 24 Unterarten den Aru-Inseln eigentümlich sind. Die nahe Verwandtschaft der Aru-Inseln mit dem südlichen NeuGuinea (Flyriver-Gebiet) ergiebt sich aus folgenden Zahlen: Vor den 171 Brutvögeln der Aru-Inseln (20 Strichvögel sind schon von der Gesamtzahl abgezogen) sind 112 Arten, ferner 21 Conspecies auch vom südlichen Neu-Guinea bekannt. Darunter findet sich allerdings eine Anzahl an und auf dem Wasser lebender Vögel (ca. $30 \mathrm{Sp}$.) von grösserer Verbreitung. Dagegen finden sich nur 52 Arten und Unterarten der Aru-Vögel auf den Kei-Inseln wieder, während 119 Arten der Vogelfauna der Aru-Inseln hier fehlen. Die für Aru charakteristischen Familien der Paradiseidae und der Casuaridae fehlen auf Kei vollkommen. Andere Familien sind dort hauptsächlich mit anderen Arten vertreten. Von den Psittaciden der Aru-Inseln fehlen 3 Gattungen auf Kei (Microglossus, Chalcopsitta, Cyclopsitta). Von der artenreichsten aller auf Aru vorkommenden Familien, den Muscicapiden mit 18 Arten ist nur eine Art und eine Unterart mit denen auf Kei identisch. Ebenso finden sich von der mit 17 Arten auf Aru verbreiteten Familie der Meliphagiden nur 2 Arten auf Kei wieder. (Im Ganzen sind 5 Meliphagiden von Kei bekannt).

Artenreich und auffallend im Landschaftsbild der Aru-Inseln sind vor allem noch die Familien der Alcediniden (11 Sp. darunter 2, die Aru eigentümlich sind, Sauromarptis tyro und Tanysiptera hydrocharis), die Columbiden (16 Sp.) und die Psittaciden (11 Sp.); andere nur durch wenige, aber weiter verbreitete Arten vertretene Familien, fallen durch ihre Individuenzahl auf, wie die Sturnidae (Mino dumonti und Callornis metallica), von den Laniiden Cracticus cassicus und die Familie der Prachtdrosseln mit Pitta atricapilla.

Eine Zusammenstellung der Vögel der Kei-Inseln hat schon früher HARTERT ${ }^{2}$ ) vorgenommen; danach sind jetzt etwa 130 Vogelarten von den Kei-Inseln bekannt. Die Vogelwelt der Kei-Inseln ist also durchaus nicht arm an Arten, wie das für einige andere Klassen zutrifft, aber die rein papuanischen Formen sind hier viel schwächer vertreten, wie aus den oben angeführten Beispielen zu ersehen ist, wie aber noch markanter aus einer Zusammenstellung von DE BEAUFORT ${ }^{3}$ ) hervorgeht (zoogeographisch bedeutungslose Arten wie Zugvögel, Seevögel u.s. w. sind dabei fortgelassen). Danach sind nur $14 \%$ der Kei-Vogelarten auch von Neu-Guinea bekannt, (von Aru 78\%), 32.5\% der Kei-Vögel zeigen Verwandtschaft zur orientalischen Region (von Aru nur $2 \%$ ), ferner ist der Prozentsatz der für die Kei-Inseln endemischen Arten $(30 \%)$ sehr viel grösser, wie der der Aru-Inseln (7\%), und schliesslich übertrifft auch die Zahl der Vogelarten mit weitem Verbreitungsgebiet auf Kei, diejenige der Aru-Inseln. Aehnliches, bezüglich westlicher und östlicher Beziehungen, gilt wie de BEAUFORT zeigt für Ceram und Halmahera, während auf den Neu-Guinea vorgelagerten Inseln Waigeu, Batanta, Salawatti und Misool, ähnlich wie auf den Aru-Inseln, das papuanische Element stark überwiegt. Die artenreichsten Familien der Kei-Inseln sind die Columbidae (14 Sp., darunter die im offenen Gelände häufige Geopelia mangei, die auf Aru fehlt), ferner die Cuculidae und Psittacidae.

56 Arten von Reptilie n sind bis jetzt von den Aru-Inseln bekannt, 34 Arten von den Kei-Inseln, wenn wir ausser der Bearbeitung von J. Roux ${ }^{4}$ ) über die Reptilien und Amphibien dieser Inseln noch spätere Veröffentlichungen berücksichtigen ${ }^{5}$ ). Von 3 Cheloniern und einem Crocodilier der Aru-Inseln

1) H. VON BERLEPSCH, Die Vögel der Aru-Inseln mit besonderer Berücksichtigung der Sammlungen des Herrn Dr. H. MERTON. Abhandl. Senckenberg. Naturf. Ges. XXXIV, 1911. - Ders. Ueber die auf den Kei-Inseln gesammelten Vogelbälge. Ebenda XXXIV, 1913.

2) E. HARTERT, On the Birds of the Key and South-Eeast Islands and of Ceram-Laut. Novitates Zoologicae VIII, 1901 und $\mathrm{X}, 1903$.

3) L. F. DE BEAUfORT, De zoogeographie van het oostelijk deel van den Indo-Australischen Archipel. Handelingen XIII. Nederl. Natuur- en Geneesk. Congres 1911. - Ders. Fishes of the eastern part of the Indo-Australien Archipelago with remarks on its zoogeography: Bijdragen tot de Dierkunde 19. Afl. 1913.

4) J. Roux, Reptilien und Amphibien der Aru- und Kei-Inseln. Abhandl. Senckenberg. Naturf. Ges. XXXIII. 1910. 5) N. DE Rooy, The Reptiles of the Indoaustralian Archipelago. I. Lacertilia, Chelonia, Emydosauria. 1915. II. Ophidia. 1917. 
mit weitem Verbreitungsgebiet ost- und westwärts können wir absehen. Fassen wir die $\mathrm{Ophidier}$ beider Inselgruppen zusammen, so können wir zunächst 8 Seeschlangenarten absondern, dann noch 3 Typhlopiden (davon eine den Kei-Inseln endemische Art, eine noch westlich der Molukken verbreitet die dritte noch von. Neu-Guinea und Australien bekannt) ausscheiden; so verbleiben noch 13 Ophidier, von denen nur eine Art auf den Aru-Inseln fehlt, während 7 Arten von den Kei-Inseln unbekannt sind. Der Artenreichtum an Schlangen ist also auf Aru grösser als auf Kei, und bezüglich der weiteren Verbreitung der Arten, finden wir, überwiegen hier wieder entschieden die Regionen von Neu-Guinea und Australien. Alle diese Ophidier haben entweder ein grosses Verbreitungsgebiet west- und ostwärts, oder finden sich nur noch in Neu-Ciuinea und Australien. Am häufigsten sieht man auf den Aru-lnseln eine weit verbreitete Nachtbaumschlange (Dipsadomorphus irregularis) und die der australischen Region angehörenden Python amethystinus und Dendrophis calligaster; auf den Kei-Inseln fällt ausserdem noch Acanthophis antarcticus durch grosse Individuenzahl auf.

Die Lacertilier sind auf beiden Inselgruppen mit sehr viel mehr Arten vertreten. Allein von der Gattung Lygosoma giebt es nicht weniger als 18 Arten auf Aru und Kei (jede Inselgruppe besitzt bisher je $2 \mathrm{ihr}$ eigentümliche Arten). Von Agamidae leben 3 Arten der Gattung Gonyocephalus mit ausschliesslich östlicher Verbreitung auf Aru und können direkt als Charaktertiere seiner Fauna betrachtet werden; neuerdings ist eine dieser Arten auch für die Kei-Inseln angegeben worden. Hier findet man noch von Vertretern dieser Familie eine östliche Physignathus-Art und 2 Arten des Genus Draco, das im westlichen Archipel heimisch ist und auf Kei, (eine Art auch auf Misool) ihre östlichste Verbreitungsgrenze erreicht $\mathrm{zu}$ haben scheint. Rechnen wir die Lacertilier beider Inselgruppen zusammen - auch die nur auf einer von beiden vorkommenden - so ergeben sich 42 Arten. Von diesen sind 19 nur noch von Neu-Guinea und Australien bekannt, 5 Arten kommen noch westlich der Molukken vor, und 9 Arten sind west- und ostwärts weiter verbreitet. (Die übrigen verteilen sich auf endemische Arten oder haben beschränkte Verbreitung innerhalb der Molukken).

Ueberblicken wir die Reptilienfauna in ihrer Gesamtheit, so ergeben sich 3 Tatsachen: 1. Die Fauna der Kei-Inseln ist ärmer an Arten als die der Aru-Inseln, 2. Die Verwandtschaft der Reptilienfauna beider Inselgruppen mit der von Neu-Guinea und Australien ist bedeutend enger als mit der entsprechenden Fauna westlicher Gebiete. 3. Auf den Kei-Inseln scheinen nach den bisherigen Forschungen mehr endemische Arten vorzukommen (4 Sp. und 2 Var.). Die unter 1 u. 3 angeführten Erscheinungen sprechen dafür; dass die Loslösung der Kei-Inseln von dem östlichen Festland früher erfolgt ist, als die der Aru-Inseln. - Andererseits zeigt die Reptilienfauna der Kei-Inseln deutliche Verwandtschaft mit der von Ceram (Acanthophis kommt dort noch vor); fast alle Kei-Schlangen sind von den Molukken bekannt, worauf besonders BARBOUR ${ }^{1}$ ) hingewiesen hat.

Ueber die Amphibie n-Fauna ist nicht viel zu sagen. Die Kei-Inseln sind an Arten und Individuen sehr arm (nur 2 Sp.). Die 9 Amphibienarten der Aru-Inseln verteilen sich auf die Ranidae (2 Sp.), die Engystomatidae (3 Sp.) und die im westlichen Archipel fehlende Familie der Hylidae (4 Sp.). Die beiden ersterwähnten Familien stammen aus der orientalischen Region, sind aber ostwärts bis Neu-Guinea, z. T. auch bis Australien vorgedrungen ${ }^{2}$ ). So handelt es sich bei den Amphibien der Aru-Inseln (auch der Kei-Inseln) im Allgemeinen um in dem Gebiet von Neu-Guinea verbreitete Formen. Eine neue Gattung und eine neue Art sind bis auf weiteres nur von den Aru-Inseln bekannt. - Rana papua und Hyla dolichopsis sind die auf Aru am häufigsten vorkommenden Arten.

Einen besonders wertvollen Beitrag zur Zoogeographie der beiden Inselgruppen lieferte MAX WEBER $^{3}$ ) in seiner Bearbeitung der Fische der Aru- und Kei-Inseln. Für tiergeographische Betrachtungen kommen nur die echten Süsswasserfische in Betracht, und allenfalls diejenigen Elemente der Brackwasserfauna, die „aus Süsswasserfischen bestehen, die einen geringen Salzgehalt des Wassers ertragen können." Dagegen sind nicht zu berücksichtigen die allgemein im Brackwasser vorkommenden Elemente und die Meeresfische, die weite Gebiete bewohnen und selbst für die Zoogeographie des

1) Th. BaRbour, A Contribution to the zoogeography of the East Indian Islands. Mem. Mus. Comp. Zool. XLIV. 1912. 2) P. N. VAN KAMPEN, De Zoogeografie van den Indischen Archipel. Bijblad Nr. 3 en 4 Natuurk. Tijdschr. Nederl. Indië, 1909 .

3) MAX Weber, Die Fische der Aru- und Kei-Inseln, Ein Beitrag zur Zoogeographie dieser Inseln. Abhandl. Senckenberg. Naturf. Ges. XXXIV. 1911. 
ganzen Indo-australischen Archipels infolge ihrer ausgedehnten Verbreitung kaum von Bedeutung sind. Von den Aru-Inseln kennt man jetzt 45 Fischarten aus dem Süss- und Brackwasser. Von diesen sind 4 Gobius-Arten ihrer Kleinheit wegen für diese Betrachtungen auszuschliessen, da sie beim Sammeln leicht übersehen werden können, sprechen aber schon für eine Zugehörigkeit zur papuanischen Region. Weiterhin sind 26 Arten aus dem Meer oder wenigstens aus dem Brackwasser bekannt. Diese Fische sind aus dem Meer eingewandert, und sind, da es für sie keine Schranke bildet, im Indischen Archipel weit verbreitet. Von den 14 verbleibenden reinen Süsswasserfischen der Aru-Inseln sind nur 2 im Indo-australischen Archipel weiter verbreitet, die übrigen 12 sind von dort nicht bekannt, ja sie fehlen schon auf den benachbarten Kei-Inseln. Diese 12 Arten sind wie. WEBER dargetan hat, durchaus australischen bezw. papuanischen Ursprungs. Zum Teil kommen die gleichen Arten, zum Teil ihnen nah verwandte Formen in dieser Region vor. Von diesen Aru-Fischen gehören 4 zur Familie der Melanotaeniinen, dieser Familie echter Süsswasserfische Australiens und Neu-Guinea's (Pseudomugil 2 Sp., Rhombatractus 2 Sp.), und 4 Arten der Gattung Eleotris sind darunter; die übrigen 4 Arten verteilen sich auf die Gattungen Neosilurus (2 Sp.), Anguilla und Bostrychus. Demgegenüber gehören die 14 Süsswasserfische der Kei-Inseln - 6 von ihnen kommen auch auf den Aru-Inseln vor - sämtlich dem Indischen Archipel an und sind z. T. sogar noch in dem indopacifischen Gebiet verbreitet. 9 von ihnen sind auch aus dem Meer bekannt, fast alle übrigen kommen wenigstens im Brackwasser vor. „Die Fischfauna des Süsswassers der Kei-Inseln hat also durchaus einen indischen Charakter.” Diese Fische sind fast sämmtlich aus dem Meer eingewandert, das für sie keine unüberwindliche Schranke bildet. So fehlt dieser Fischfauna "jede Beziehung zu den für die Aru-Inseln, Neu-Guinea und Australien charakteristischen Süsswasserfischen."

Aehnliches gilt für die allermeisten Süsswasserfische der zwischen Neu-Guinea und den grossen Sunda-Inseln gelegenen Inseln. Echte Süsswasserfische fehlen ihnen; die Cyprinidae sind beispielsweise nicht weiter wie Lombok ostwärts vorgedrungen. Die Süsswasserfische des ganzen mittleren Uebergangsgebiets des Archipels sind aus dem Meere eingewanderte Formen, während wiederum die NeuGuinea unmittelbar vorgelagerten, oben bei Besprechung der Avifauna erwähnten Inseln, soweit bekannt, ähnlich den Aru-Inseln ausschliesslich Elemente der australischen Region in ihrer Fischfauna aufweisen.

Von anderen Abteilungen der Süsswasserfauna sind für diese Betrachtungen noch die Decapoden und Schnecken zu berücksichtigen. Wie J. Roux ${ }^{1}$ ) in seiner Bearbeitung der Süsswasser-Decapoden nachweist sind in zoogeographischer Hinsicht nur die Parastaciden von Interesse. Die Vertreter der übrigen für die Aru- und Kei-Inseln nachgewiesenen Familien kommen infolge ihrer weiteren Verbreitung hierfür nicht in Betracht. Die Parastaciden sind hauptsächlich von Australien bekannt. Die auf den Aru-Inseln vorkommende Art Cheraps quadricarinatus - auf den Kei-Inseln ist sie unbekannt bewohnt Nord-Australien und Neu-Guinea. Sie fand sich beispielsweise auch als einziger Parastacide in den Sammlungen, die H. A. LoRENTZ von seinen Reisen aus Südwest-Neu-Guinea mitgebracht hat ${ }^{2}$ ),

Sehr viele tropische Süsswasser-Mollusken sind, ähnlich wie die Fische, nicht auf das Süsswasser beschränkt und können verhältnismässig salziges Wasser vertragen: auch wandern viele marine Formen in das Brackwasser ein und können bis in das Süsswasser vordringen. Auf den AruInseln finden sich durch Sungis und Seitensungis (siehe meinen Reisebericht ${ }^{3}$ ) ständig alle Uebergänge zwischen Meer- und Süsswasser, und so bietet sich für die Mischung der beiderseitigen Faunen gute Gelegenheit. Daraus erklärt sich auch wohl (ich stütze mich in diesem Abschnitt auf C. BoETTGERS Bearbeitung ${ }^{4}$ ) z. T. die weite Verbreitung vieler Süsswasserformen (wie vieler Melanien und Neritinen). Von zoogeographischer Bedeutung ist das Physiden-Genus Isidora, das mit einer auf den Aru-Inseln neugefundenen Art sich dem Formenkreis der von Neu-Guinea bekannten Art anschliesst, während andere Arten von den Kei-Inseln dem westwärts verbreiteten Formenkreis angehören. Ebenso ist eine neue Subspecies einer in Neu-Guinea verbreiteten Vivipara auf Aru gefunden worden. Von Lamelli-

1) J. Roux, Susswasserdekapoden von den Aru- und Kei-Inseln. Abhandl. Senckenberg. Naturf. Ges. XXXV. 1919.

2) J. Roux, Crustacés, (Exped. 1907, 09 et โ2). Nova Guinea. XIII. 1921.

3) H. Merton, Forschungsreise in die südöstlichen Molukken Aru- und Kei-Inseln. Abhandl. Senckenberg. Naturf. Ges. XXXIII. 1910.

4) C. R. BoETTGER, Die von Dr. MERTon auf den Aru- und Kei-Inseln gesammelten Wassermollusken. XXXV. 1918. Abhandl. Senckenberg. Naturf. Ges. 
branchiaten ist eine neue Sphaerium-Art für die Aru-Inseln bemerkenswert. Diese Gattung fehlt im Indo-australischen Archipel; wohl aber sind Sphaerienarten. aus Deutsch-Neu-Guinea und aus Australien bekannt. Diese wenigen charakteristischen Formen beweisen, dass bezüglich der Wasser-Mollusken die Aru-Inseln - aber nicht mehr die Kei-Inseln — dem papuanisch-australischen Kreis angehören.

Viel ausgeprägter ist aber das Bild, das uns die Landschneckenfauna zeigt. Etwas beeinträchtigt die Klarheit des Bildes, dass die Molluskenfauna der Kei-Inseln weniger gut bekannt ist, als die der Aru-Inseln. Von den 34 Arten, die man nach BoETTGER ') jetzt von den Aru-Inseln kennt, sind wegen ihrer weiteren Verbreitung 3 Arten (eine davon infolge Verschleppung durch den Menschen) auszuschalten. Ueberraschend gross ist die Zahl der bisher nur den Aru-Inseln eigentümlichen Arten und Unterarten. BoETtGer ist der Ansicht, dass von den 14 endemischen Arten sicher die meisten auf Neu-Guinea vorkommen, aber bisher wegen der noch verhältnismässig geringen Erforschung dieses Landes von dort noch nicht bekannt sind. Eine nahe Verwandtschaft zu Neu-Guinea-Formen muss für die meisten dieser Arten angenommen werden, und das Gleiche gilt für 5 von 8 den Aru-Inseln eigentümlichen Unterarten. Die 3 übrigen Subspecies gehören Arten an, die im Indo-australischen Archipel weit verbreitet sind. Die Zugehörigkeit zur papuanischen Region zeigen noch deutlicher zwei Unterarten der beiden Arten Xesta citrina und Leptopoma vitreum, die im Indo-australischen Archipel weit verbreitet sind und "in den meisten Gebieten Subspecies und gute Lokalformen ausbilden”. Auf den Aru-Inseln leben die gleichen Unterarten wie auf Neu-Guinea. Ferner sind noch 7 richtige Arten beiden Gebieten gemeinsam.

Von den weniger erforschten Kei-Inseln kennt man erst 7 Arten sicher, z. T. aber auch deshalb, weil die Molluskenfauna dieser Inseln sehr viel ärmer ist, als die der benachbarten Aru-Inseln. Von den 7 Arten ist eine nicht verwertbar, da sie der Adventivfauna angehört. Auf den Kei-Inseln leben von den beiden oben mit Namen erwähnten Arten andere Unterarten, als auf den Aru-Inseln; diese KeiSubspecies stehen am nächsten anderen Unterarten der Tenimber-Inseln und von Amboina. Weiterhin ist eine im westlichen Archipel verbreitete und bis zu dem gleichen Inselbogen vorgedrungene Art auf den KeiInseln mit einer besonderen Unterart vertreten. Die drei übrigen den Kei-Inseln eignen Arten gehören zu Gattungen, die auf den Aru-Inseln mit anderen Arten vertreten sind. - Die Funde an Nacktschnecken können vorderhand das Bild nicht vervollständigen. - Auffallend ist schon jetzt für die Kei-Inseln das Fehlen einer ganzen Reihe häufiger Charakterschnecken der Aru- und Neu-Guinea-Fauna und ebenso bemerkenswert ist es, dass die Landschneckenfauna der Kei-Inseln eine grössere Verwandtschaft zeigt zu der benachbarten Amboina-Gruppe und den Tenimber-Inseln, als zu den Aru-Inseln.

Schliesslich möchte ich an Hand der Bearbeitung von PAGENSTECHER ${ }^{2}$ ) über die Schmetterlinge der beiden Inselgruppen hier noch einige Angaben machen. Von 230 Arten von Tagschmetterlingen - die Nachtschmetterlinge sind noch nicht ausreichend bekannt, tiergeographisch auch weniger wichtig - sind nur $57 \mathrm{Sp}$. beiden Gebieten gemein, und davon haben wiederum etwa $2 / 3$ ein weites Verbreitungsgebiet. Der papuanische Charakter der Schmetterlingsfauna der Aru-Inseln ist deutlich ausgeprägt; 7 Gattungen von Tagschmetterlingen (mit mehreren Arten) der Aru- und Neu-Guinea-Fauna fehlen vollständig auf den Kei-Inseln. Ueberhaupt fällt auf den Kei-Inseln das papuanische Element viel weniger auf. Neben einer Reihe' endemischer Formen und solchen, die nur von den Aru- und Kei-Inseln bekannt sind, halten sich Formen mit westlichem und solche mit östlichem Verbreitungsgebiet ungefähr die Wage. Eher überwiegen auch hier noch die papuanischen Formen; dies scheint wenigstens aus der Zusammenstellung von PAGENSTECHER hervorzugehen. Allerdings ist dabei zu berücksichtigen, dass er Ceram zur papuanischen Region rechnet, was ja auch im Hinblick auf andere Abteilungen (z. B. kommt dort noch der Casuar vor) z. T. seine Berechtigung besitzt.

Die Einzeluntersuchungen über die zoogeographisch wichtigen Tiergruppen der Aru- und KeiInseln stimmen in ihren wesentlichen Ergebnissen gut überein, wenn auch zunächst ein gewisser Unterschied in der Verbreitung zwischen den Formen der Land- und Süsswasserfauna zu bestehen scheint. Bei genauer Betrachtung ergiebt sich aber, dass erst durch die Kenntnis der Zusammensetzung

1) C. R. Boettger, Die Landschneckenfauna der Aru- und der Kei-Inseln. Abhandl. Senckenberg. Naturf. Ges. XXXV. 1922.

2. A. PAGENSTEChER, Die Lepidopteren der Äru- und Kei-Inseln. Abhandl. Senckenberg. Naturf. Ges. XXXII. 1911. 
der Land- und der Süsswasserfauna ein klares Bild der Zoogeographie dieser beìden Inselgruppen gewonnen werden kann. Das papuanische (z. T. auch australische) Element, das auf den Aru-Inseln dominiert, ist auf den Kei-Inseln schon starkabgeschwächt vorhanden; das zeigt deutlich ein Vergleich der Beuteltier-Fauna der beiden Inselgruppen, das geht ebenso klar aus einem Vergleich der beiden Avifaunen hervor. Die Reptilien und Amphibien beweisen es nicht minder, und auch die Landschnecken, selbst die Tagschmetterlinge können dafür angeführt werden. Es haben also viele Aru-Formen die Kei-Inseln, vor deren Loslösung vom östlichen Feștland, nicht mehr erreicht.

Andererseits sind die Ausstrahlungen der orientalischen Region auf den KeiInseln in verschiedener Stärke in den einzelnen Tiergruppen wahrnehmbar. Wir dürfen dabei nicht vergessen, dass die indische Fauna von ihrem Ausstrahlungszentrum einen viel weiteren Weg zurückzulegen hatte, als die papuanischen Formen, dass dieser Weg offenbar nur ganz vorübergehend benutzbar war, und dass schon viel weiter westlich gelegene Inseln eine stark verarmte indische Fauna aufweisen. Daher darf es nicht Wunder nehmen, wenn auf diesen äussersten Vorposten der orientalischen Region im allgemeinen die indischen Formen höchstens der papuanischen Komponente die Wage halten. Von Reptilien überwiegt auch hier noch bedeutend der östliche Einfluss. Auf den Aru-Inseln ist in den zoogeographisch wichtigen Tiergruppen nur noch ein ganz geringfügiger Prozentsatz indischer Formen nachweisbar. Viel auffallender ist der Gegensatz zwischen den Landschnecken der Aru- und Kei-Inseln. Das kommt zwar nicht zahlenmässig zum Ausdruck, aber die markanteren Formen der Aru-inseln sind durchaus papuanisch, diejenigen der Kei-Inseln zeigen nahe Verwandtschaft zu denen der Amboina-Gruppe und der Tenimber-Inseln und reihen sich an die Formenkreise der orientalischen Region. Aehnliches gilt für die Süsswasser-Mollusken, und am auffallendsten wird diese Verschiedenheit bei Betrachtung der echten Süsswasserfische. Auf den Aru-Inseln leben die echten Süsswasserfische der papuanisch-australischen Region. Sie fehlen sämtlich auf den Kei-Inseln. Stattdessen leben hier weitverbreitete Formen der orientalischen Region, die ursprünglich aus dem Meer eingewandert sind.

In der Gruppe der Süsswasserfische erreicht die Verschiedenheit zwischen der Tierwelt der Aru- und der Kei-Inseln ihren Höhepunkt. Wenn auch die verschiedenartige Beschaffenheit der Süsswasserläufe auf den meist flachen Aru-Inseln und in den reissenden kurzen Gebirgsbächen von Gross-Kei sehr verschiedene Bedingungen bieten, so ist damit doch nicht erklärt, warum Fische papuanischen Arsprungs hier vollkommen fehlen. Nur eine weiter zurückliegende Lostrennung der Kei-Inseln von dem gemeinsamen östlichen Festland lässt diese Verschiedenheit der Fischfaunen plausibel erscheinen. Erinnern wir uns andererseits daran, dass die Kei-Inseln allein von allen Molukken noch 5 Beuteltier-Arten besitzen (von den Aru-Inseln kennt man 9) darunter, wie WEBER ${ }^{1}$ ) sich ausdrückt eine so moderne Form wie Macropus (= Thylogale), so verbietet uns dieses Vorkommen die Loslösung der Kei-Inseln weit in das Tertiär zurückzuverlegen. Wir haben damit zwei Angaben, die, wenn die geologische Geschichte dieser Gebiete besser bekannt sein wird, dazu verhelfen können den Zeitpunkt der Loslösung der Kei-Inseln genauer zu bestimmen. Gleichzeitig wird man darüber Aufschluss erhalten, innerhalb welches Zeitraums die Ausbreitung der Arten erfolgt ist und wird weiter daraus schliessen können, wie gross etwa der Zeitabschnitt war, der in den einzelnen Gruppen zur Bildung neuer Arten führte.

Die Schlussfolgerungen, die sich aus dieser Zusammenstellung zur Beurteilung der Zoogeographie der Aru- und der Kei-InseIn klar ergeben, werden noch gesichert und gestützt, wenn wir die ozeanographischen und geologischen Tatsachen berücksichtigen. Die Aru-Inseln erheben sich aus der Flachsee, die sich zwischen Australien und Neu-Guinea ausdehnt. Eine Niveauveränderung von $50 \mathrm{~m}$. genügte um das Festland, das Australien, Neu-Guinea und die Aru-Inseln umfasste in durch das Meer getrennte Gebiete zu verwandeln. Unter Zugrundelegung der PENCK'schen Anschauungen hat MAX WEBER ${ }^{2}$ ) für den Indo-australischen Archipel klar entwickelt, dass durch eine Hebung der Strandlinie um $70 \mathrm{~m}$., wie sie als Folge des Abschmelzens der Eismassen im Pleistocän angenommen wird,

1) MAX Weber, Der Indo-Australische Archipel und die Geschichte seiner Tierwelt. Jena 1902.

2) MAX WEBER, Neue zoogeographische Probleme aus dem Indo-australischen Archipel. Festschr. f. MAX FüRBRINGER. Heidelberg, 1919. 
im östlichen und westlichen Archipel eine Trennung der Landmassen herbeigeführt wurde, die bis dahin dem australischen bezw. dem asiatischen Kontinent angehörten. Der spättertiäre australische Kontinent umfasste mithin, wie sich z. B. aus der Betrachtung der hydrographischen Karten der SibogaExpedition entnehmen lässt, ausser Australien und Neu-Guinea, noch die Neu-Guinea westlich vorgelagerten Inseln Waigeu, Batanta, Salawatti, Misool und die Aru-Inseln, die jetzt alle durch die Flachsee von Neu-Guinea getrennt sind. Das Vorherrschen des papuanischen Elements in der Fauna dieser Inseln ist darum gut verständlich. Der übereinstimmende Charakter der Süsswasserfauna dieser Gebiete erklärt sich zum Teil zwanglos „durch den einstmaligen Zusammenhang heutzutage getrennter Süsswassersysteme" (WEBER p. 11), indem Flüsse jetzt getrennter Gebiete, Nebenflüsse eines grösseren Systems waren.

Zwischen den Aru-Inseln und den $125 \mathrm{~km}$. westwärts davon gelegenen Kei-Inseln, die von Neu-Guinea nur wenige $\mathrm{km}$. weiter entfernt sind, als die Aru-Inseln, liegt ein tiefer Graben mit Tiefen bis zu $3565 \mathrm{~m}$., wie durch die Siboga-Expedition nachgewiesen worden ist. Dieser Umstand scheint dafür zu sprechen, dass die Trennung der Kei-Inseln von Neu-Guinea und den Aru-Inseln schon weiter zurückliegt, und die Zusammensetzung der Fauna der Kei-Inseln bestätigt diese Vermutung. Dem gegenüber lassen sich aber z. B. unter den Beuteltieren, die auf beiden Inșelgruppen vorkommen keinerlei Unterschiede feststellen. Man darf also nicht entsprechend der grossen Meerestiefe zwischen den beiden Inselgruppen und zwischen Kei und Neu-Guinea die Loslösung der Kei-Inseln weit in das Tertiär zurückverlegen. Es handelt sich hier um einen Einbruchsgraben, der erst gegen Ausgang der Tertiärs entstanden sein kann. Die Untersuchungsergebnisse dieser Forschungsreise sind somit wohl geeignet, diese schon früher von WEBER vertretene Auffassung zu stützen.

Im übrigen können wir aber aus den jetzigen Meerestiefen in diesen Gegenden unschwer die Landbrücken erkennen, auf denen die Tierwelt der orientalischen Region zu den Kei-Inseln vorgedrungen ist. Die Kei-Inseln liegen an der östlichsten Stelle des Inselbogens, der von Amboina und Ceram nach den Tenimber-Inseln zieht und der die Banda-See nach Osten abgrenzt. Die Meerestiefen zwischen den einzelnen Inselgruppen des Bogens bewegen sich zwischen 200 und $800 \mathrm{~m}$. Die Kei-Inseln sind also nach allen Seiten von Meeren mittlerer Tiefe umgeben, woraus geschlossen werden kann, dass sie schon länger als die Aru-Inseln nicht mehr durch irgend eine Landbrücke mit Nachbargebieten verbunden waren. Die Untersuchungen über die Fauna der Kei-Inseln bestätigen dies. Auf den Kei-Inseln finden wir mehr endemische Arten als auf den Aru-Inseln. Andererseits sind viele KeiFormen in den Molukken weiter verbreitet, und es ist wohl möglich, dass die Kei-Inseln nach ihrer östlichen Loslösung noch eine Zeitlang mit benachbarten Inseln des Molukkenbogens in Verbindung gestanden haben.

Aus der Zusammensetzung der Fauna der Kei-Inseln können wir noch einen anderen Schluss ziehen. Obwohl die Kei-Inseln voraussichtlich mit Neu-Guinea in Verbindung standen, als dies noch mit Australien verbunden war, ist doch das östliche Element in der Kei-Fauna überwiegend papuanisch. Die Kei-Inseln haben also - entsprechendes gilt für die Aru-Inseln — wie das schon aus ihrer Lage hervorzugehen scheint, länger mit Neu-Guinea in Verbindung gestanden als mit Australien, denn von Ausnahmen abgesehen, sind alle Tierarten, die auf den Kei-Inseln und in Australien vorkommen auch von Neu-Guinea bekannt, aber umgekehrt fehlen viele Formen in Australien, die auf Neu-Guinea und auf den Kei-Inseln vorkommen.

Die speziell geologischen Verhältnisse der Aru- und Kei-Inseln sind noch wenig geklärt. Das flache Massiv der Aru-Inseln, das von einer Reihe in hauptsächlich in west-östlicher Richtung verlaufenden Haupt-Sungis (Seewasser-Kanälen) in mehrere Inseln geteilt wird, besteht zum grossen Teil aus quartären Kalk- und Sandsteinen. Einzelne Funde machen ein tertiäres Alter, wenigstens eines Teils der Inseln wahrscheinlich. Die in einer Gesteinsprobe aus dem inneren Terangan, der südlichsten der Aru-Inseln, nachgewiesenen Orbitoiden sprechen nach VERBEEK ${ }^{1}$ ) für die Zugehörigkeit zum OberMiocän, und auch auf den Aru-Inseln gefundene fossile, keulenförmige Cidaris-Stacheln und eine Pecten lassen auf ein höheres als quartäres Alter der betreffenden Schichten schliessen ${ }^{2}$ ).

1) R. D. M. VERBEEK, Bemerkungen über die von den Aru-Inseln mitgebrachten Gesteinsproben. Im Anhang zu meinem Reisebericht. Abhandl. S. N. G. XXXIII. p. 206.

2) L. DöDERLEIN, Ueber Echinoidea von den Aru-Inseln. Abhandl. S. N. G. XXXIV, 1911. 
Die Haupt-Insel der Kei-Gruppe, Gross-Kei, in etwa nordsüdlicher Richtung verlaufend, besteht aus einer schmalen bis zu $800 \mathrm{~m}$. ansteigenden Gebirgskette; die gleichen von hier bekannten Alveolinenkalke sind auch auf Neu-Guinea gefunden worden und sprechen für eine frühere Landverbindung. Auch Gesteine eocänen Alters sind von Gross-Kei bekannt. Die weiter westlich gelegenen Inseln von Klein-Kei sind flach und bestehen aus grobkörnigem Korallenkalk; sie haben sich sehr wahrscheinlich erst im Quartär über das Meer erhoben.

Bei eingehenderer Kenntnis der Fauna der Aru- und der Kei-Inseln werden sich vielleicht noch Unterschiede in der Besiedelung der grösseren Inseln innerhalb jedes dieser beiden Archipele ergeben. Das gilt vor allem für Gross- und Klein-Klei, die mit ihrer verschiedenartigen Bodengestaltung und ihrem unterschiedlichen Wasserreichtum - Klein-Kei ist sehr arm an Süsswasser, Gross-Kei besitzt kurze Gebirgsbäche von starkem Gefälle - andere Existenzbedingungen bieten.

Durch diesen Einblick in die Zusammensetzung der Faunen dieser beiden östlichsten Inselgruppen, sind wir in der Lage das Bild der Tierverbreitung im östlichen Indo-australischen Archipel zu vervollständigen. Es erübrigt sich hier auf die Zoogeographie des ganzen Archipels näher einzugehen. MAX WEBER, ein hervorragender Kenner des Archipels, hat in Einzeluntersuchungen und zusammenfassenden Arbeiten in mustergültiger Weise diese ganzen Fragen in ihren Zusammenhängen behandelt.

Das Indo-australische Uebergangsgebiet, das östlich der grossen Sunda-Inseln beginnt, endigt auch im Osten nicht mit einer scharfen Grenzlinie. Je nach der Tiergruppe, die wir der Betrachtung zugrunde legen, muss die Grenze verschieden verlaufen. Die nordwestliche Grenzlinie des australischen Kontinents ist wohl zu Ausgang des Tertiärs - DE. BEAUFORT hat auf Grund der Verbreitung der Vögel auch schon darauf hingewiesen - westlich von Waigeu und Misool, in südöstlicher Richtung umbiegend, dann östlich von Ceram, $z$ wischen den Aru- und Kei-Inseln verlaufen. Sie stimmt mit der $200 \mathrm{~m}$. Tiefenlinie des Meeresbodens gut überein. - Tiergeographie und Erdgeschichte ergänzen sich hier gegenseitig und erklären die Verschiedenartigkeit in der Zusammensetzung der Tierwelt der Aru- und der Kei-Inseln.

Es ist mir eine besondere Freude dem sehr verehrten Jubilar, Herrn Prof. MAX Weber, der mit grossem Interesse den Verlauf und die Ergebnisse meiner Forschungsreise verfolgt hat, sowie selbst lebhaften Anteil hat an der Auswertung ihrer Ausbeute, als Zeichen aufrichtigen Dankes diese kleine Zusammenfassung in herzlicher Verehrung widmen zu dürfen. 